\title{
The Benefits of Teaching Yoga to Young Children with Special Needs: Developing an Appropriate Methodology
}

\author{
Michelle Mochan \\ Laurentian University, Canada
}

\begin{abstract}
Yoga has many benefits for children and has been shown to improve health and well-being. Yoga can be used to improve the academic performance of all students and is especially valuable as an intervention method for students with special needs.

Despite the benefits that yoga can have for young children there are barriers that prevent teachers from using yoga in their classroom. However, the concerns over meeting the curriculum, lack of space and maintaining student engagement can be overcome by incorporating yoga into the curriculum, being creative with the space that is available, using age appropriate teaching methods (such as games) and limiting the duration of the session to better suit the capabilities of the students.

However, much of the research on the effects of teaching yoga to young children use methodologies that are unsuited for that particular age group. The purpose of this study is to contribute to the research by implementing a more effective methodology. Furthermore, this study is of practical use to teachers as it discusses how yoga can be brought into the classroom and integrated with the curriculum thus increasing the time that is available for teaching.
\end{abstract}

\section{Introduction}

Teachers have been asked to keep up with the growing needs of the students in their classrooms, as the number of students with special needs in classrooms increases yearly. Frequently children with special needs display unique behaviours that require additional classroom management. It is at the crossroads between teaching, accommodating students with special needs, and classroom management, where incorporating yoga into the day becomes beneficial for teachers [1], [2], [3].

Numerous papers focus on the benefits of yoga for students but treat yoga as something that should be added to the day separate from everything else, or as something that can be added onto physical education [4]. However, few papers discuss how yoga can be incorporated into the typical school day [5]. Furthermore, when mindfulness has been used as in a school setting it was not always implemented by the classroom teacher thus it is difficult to determine whether mindfulness can be used effectively by the regular classroom teacher [6].

Additionally, few studies are conducted in an elementary school and fewer still focus on young children and preschoolers [2, 7]. The appropriateness of yoga is called into question. Recent systematic reviews of the literature concur that the benefits of yoga to young children are inconstant primarily due to issues of appropriateness in the methods [7], [8]. Meaning that the yoga sessions were unsuitable for children either due to their length, inappropriate testing (asking young children to complete a battery of cognitive tests, or relied solely on self-report measures), or lack of control for confounding variables, such as drugs [2], [4], [8]. Where the duration of individual sessions was listed the studies that did not find yoga to be beneficial were often an hour or longer, with some sessions with four to six year olds being two hours long [7, 8].

The aim of this paper is threefold. First, this paper will review the literature in order to establish how teachers might effectively use yoga to support students with diverse learning needs. Secondly, this paper examines existing research, in conjunction with the Ontario curriculum in order to establish how yoga can be incorporated into the curriculum. Finally, this study examines how yoga can be taught to young children between the ages of 4 to 8 , with the intention of determining which methods are most appropriate for teaching yoga to young children.

Yoga is a Hindu philosophy that teaches people to control their body and mind in order to reach inner peace [9]. Traditionally yoga was a way to unite the physical, spiritual, and emotional aspects of a person, thereby allowing them to reach a state of inner peace, or mindfulness [9]. Yoga has three components: the emotional (breath), the physical (poses), and the spiritual (meditation) [9]. Yoga consists of many different subtypes which focus more heavily on certain aspects compared to other components.

Physically, yoga improves balance, flexibility, coordination; helps develop kinesthetic awareness, 
and motor skills [9]. Emotionally, yoga helps children recognize and control their emotions and improve emotional regulation, which helps develop empathy and provides them with tools to calm down if they are upset or angry [2]. Socially, yoga improves relationships among peers by building a community of mutual trust and respect; children who can identify and explain their emotions are better able to communicate with others [2], [3]. Developing empathy fosters a greater understanding and appreciation for their peers, thus fostering stronger social ties [3]. Mentally, yoga improves concentration, focus, and creativity. The development of such skills fosters resilience and teaches children the coping skills necessary to encourage mental wellbeing. Thus, yoga allows children to explore and develop their identity [9].

In Canada, the average person starts school at age 6 with the option to start kindergarten at the age of 4 , the school day lasts approximately 6 hours with students spending a minimum of 12 years in school. As a result, school is one of the primary means of socialization and has a significant role in shaping people's lives [8]. Consequently, schools have the ability to teach students about health and resilience early so they can form meaningful lifelong habits that positively impact their health over their lifespan $[8,9]$. If students are to learn they must be healthy both mentally and physically; and research has long established that health and academic performance are closely related [9]. Therefore, it is crucial to develop and investigate programs that improve the overall health and development of children.

\section{Literature Review}

Various articles cite the physical and mental benefits of yoga. Yoga is a means for improving students' academic performance, concentration, and attention, which help children learn and retain new information [1], [2], [5], [6], [8]. Some studies suggest that yoga can improve the blood flow to the frontal lobe, thus improving executive functioning [8]. Low executive functioning is a main component of many developmental and learning disabilities [1], [7], [9]. Improving executive functioning improves concentration and memory, boosting the academic performance of all students [2], [5], [8].

The greatest barrier to implementing yoga in the classroom is the pressure to teach to the curriculum and the lack of time teachers have available [6]. This barrier can be overcome by integrating yoga into the curriculum. Yoga can be integrated into the curriculum to facilitate teaching movement competence and emotional regulation, two key aspects of the physical education and health curriculum $[2,10]$. Nevertheless, yoga is not limited to meeting the expectations of the health curriculum. Depending on how the lessons are designed yoga can meet a variety of curriculum expectations in different subjects across both the kindergarten and primary curriculum.

Yoga can also be used as a classroom management tool, in addition to setting the tone of the class. A study by Hassar found that yoga had a substantial effect on establishing the atmosphere of the classroom [6]. Yoga was beneficial for regaining the attention of students and resetting the classroom environment and tone in a comparatively short period of time [6]. Yoga can create a calm and peaceful environment and refocus student's attention towards a more focused lesson when transitioning between subjects or after breaks [6]. Many students struggle during times of transitions, especially children with conduct disorders, Autism and ADHD as they are typically a time when a classroom becomes chaotic [6]. Yoga is of particularly beneficial tool for classroom management during this time as it can help ease the tone of the class into a more peaceful state, allowing teachers to continue with the lesson more readily [6].

Students need to be taught the foundations of mindfulness at an early age, to allow these practises to become tool that foster resilience. The earlier students learn these practices, the more they benefit in the future.

\subsection{How yoga meets diverse learning needs}

Research suggests that yoga can be used to help children diagnosed with Autism Spectrum Disorder (ASD) [4], [5], [7]. Yoga is uniquely suited to help children with ASD learn social skills as it was found that students with ASD are more receptive to interventions which place an emphasis on movement and sound [4]. As yoga incoprtate repetitive movement which is proven to calm children with ASD, this study suggests that yoga interventions could result in a higher level of engagement when compared to more traditional interventions for Autism and thus may be more effective [4].

Furthermore, children with ASD often interpret input from muscles easier than other forms of sensory input (e.g. hearing, sight) and as such may have an easier time learning yoga [4]. Developing language and social skills early is critical for any child and has beneficial long-term effects on the outcomes for children, particularly those affected by ASD.

Yoga is often taught through imitation, which is a critical skill for all children and one that many children with Autism lack; continuous practice of 
imitation in young children will improve language outcomes, and social skills [4]. A study conducted by Porters, J (2013) found that children with Autism who participated in daily yoga sessions used more verbal communications, socialized more with peers and made more eye contact then they did before starting the yoga intervention [4].

Yoga has proven to reduce the severity of both anxiety and depression through the practice of mindfulness [6].Yoga has been shown to increase in the levels of $y$-amino butyric acid (GABA) in the brain by as much as $27 \%$. Low levels of GABA are associated with depression and anxiety thus; increasing the levels of GABA in the brain through yoga may reduce the severity of these disorders [5].

Poses, breath work and meditation are all important aspects of the physiological mechanisms of yoga and work in connection with each other to produce beneficial effects [11]. The combination of breath work, postures and meditation undertaken in yoga are important in counteracting the stress response [6]. Physical poses stimulate the digestive process and increase blood flow to the organs, reducing the physical symptoms of anxiety and depression and thus may have a more long-term antidepressant effect [11]. While breath work calms the sympathetic nervous system and increased activation of the parasympathetic nervous system resulting in an increased emotional self-regulation [6], [11]. The meditation decreases negative cognitions which contribute to depression and anxiety and increases mindfulness.

Mindfulness encourages students to be aware of their thoughts and therefore can help students identify negative thoughts and ruminations and eventually reduce the prevalence of ruminations [6]. Ruminations and negative thought patterns such as catastrophizing have been linked to the persistence of depression and anxiety [6].

Decreasing the amount of negative thoughts, as well as decreasing stress is proven to help children sleep, therefore Yoga can also be used to assist children who suffer from sleep disorders [6].

Research concurs that yoga is useful for treating children with disorders such as attention deficit hyperactivity disorder (ADHD) as mindfulness practices increase focus and the ability to delay gratification and reduces impulsivity [9]. Yoga, in particular is easier for children with ADHD to practice and benefit from, as it incorporates movement and does not require that students sit still for long periods, this is especially necessary for children who struggle with hyperactivity [6].

Additionally yoga eaffects brain structure and neurochemistry by reducing cortisol and increases serotonin and dopamine thereby reducing stress and improving the mood of students [12]. As atypical attention spans and impulsivity are major symptoms of ADHD, these studies provide compelling empirical evidence for using yoga exercise in ADHD treatment $[6,12]$.

Mindfulness strengthens the student's ability for the self-regulation of attention, which is crucial for learning. The ability to self-regulate their attention is a key component of ADHD and thus practise that can help these children increase the attention span is especially beneficial for students with ADHD [6]. Attentional control is developed by teaching children how to focus, sustain, and shift attention mindfully; through breath work and guided meditation [6]. The literature supports the findings that mindfulness is a constructive means of supporting students with ADHD [6].

\subsection{Yoga as a classroom management tool}

The management of behavior in a classroom considered a cornerstone of teaching [13]. Classroom management is about building a tone of respect in order to create collaborative communities in which students feel safe and supported. Students who struggle to manage their emotions as well as their behavior often do not feel a sense of support in their classroom [13]. This makes it difficult for these students to function in a group, and interferes with learning. Yoga allows students to move and be active while maintaining a relaxed state, giving children the confidence to express themselves in a safe, supportive classroom [13]. Yoga helps students to calm down, get centered, so that they can then reconnect, either with other students, or with their learning; while allowing teachers to benefit.

By participating in the yoga with their students' teachers also slow down their breathing, feel centered, and present [13]. This means that teachers are able they are to teach and to deal with challenging situations with greater ease. Albrecht, Albrecht and Chohen found that teachers who participated in yoga with their students taught in a less disjointed fashion and were able to increase the depth of knowledge they imparted in their lessons [14]. Additionally, it was found that teachers were able to identify key concepts they in their lessons with greater ease, allowing them to teach with greater clarity [14]. This improvement in their communication style in turn meant that students grasped new material and key concepts faster [14]. Teachers generally reported feeling less overwhelmed by the demands of the curriculum and the learning objectives they were required to complete [14].

Yoga's primary use as a tool for classroom management is through teaching self-regulation [2, 9]. Teaching children to self-regulate at an early age means that teachers spend less time managing student behavior; as children learn to control the 
emotions that cause them to act out; leaving more time for teaching [2]. Implementing mindfulness techniques reduces fatigue, and stress as well as improving metacognition and teaching children to control their instinctive flight-or-flight reactions by suppressing the limbic system [6]. This allows children to analyse conflict more easily and leads to better problem solving; thus resolving situations before it create conflict in the classroom [6].

Mindfulness allows children to become aware of emotions and behaviors. Recognizing their emotions and behaviors is the first step to controlling them [2]. Additionally, meditation and deep breathing exercises are commonly used as a way to deal with a variety of emotions such as anger, frustration, sadness, and anxiety. Children and teachers can only benefit when students learn how to calm down when they feel themselves becoming upset or frustrated [2]. If students can understand mindfulness practices at an early age, it becomes a tool which can be used to reduce behavioral outbursts in the future.

\subsection{Barriers to teaching yoga in the classroom}

There is a wealth of existing research on how yoga can be used to teach students with special needs, improve emotional regulation, and foster academic success [1], [2], [4], [5], [6], [7], [8], [9], [11], [12]. However, students will not receive the benefits of yoga if teachers do not use it in their classrooms. Therefore, it is necessary to examine what barriers prevent teachers from practising yoga with their students and discuss ways to mitigate these barriers. A review of the literature has found that there are three main barriers that prevent teachers from implementing yoga in a classroom environment: the demands of the curriculum, student engagement, and a lack of space [6], [14].

The lack of physical space in the classroom is in some ways the most difficult barrier to overcome; teachers cannot expand the physical size of a classroom to suit their lessons. Instead, the lessons need to be adapted to the space that is available. However, there is no reason that yoga cannot be practised outside or in the gymnasium in so far as schedules and weather permits. For times when this is not feasible other solutions must be found. Rearranging the furniture in a classroom can only open up so much space; therefore the yoga must be modified to suit the environment. Many yoga poses can be modified to be done in a sitting or standing position that does not require much space. Chair Yoga is a type of yoga that can be done entirely while seated in a chair, making it an ideal type of yoga for classrooms where limited space is available. Additionally there are many games that require students take turns, this would limit the number of people performing yoga at a time, thus requiring less space than if all the students were performing the poses at once.

Maintaining student engagement is another barrier that prevents teachers from brining Yoga into the classroom. In a study by Harssar by teachers expressed that they needed to regularly design and employ different creative activities for their students to partake in. Teachers stated that students often became disinterested when they repeat the same poses or type of meditation every day [6]. Harssar found that students responded best to activities that were similar to games [6].

The struggle to maintain a student's engagement in a lesson, or subject is not a common difficulty that all teachers encounter and must learn to overcome; it is not limited only to teaching yoga. New yoga techniques and novel poses are one way of maintaining interest, so is making yoga into a game or finding way ways to make yoga relevant to the students that are being taught. Additionally asking students to get involved in planning the lesson; either by picking what yoga poses they want to practise, or allowing them the creativity and independence to create their own poses can also increase and maintain student engagement.

The final barrier to teaching yoga in the classroom is time constraints and the pressure to cover the curriculum. This problem can be solved by incorporating yoga into the curriculum. Yoga can be incorporated into the curriculum in a variety of ways, the following section suggests a few ways, but by no means is yoga limited to these subject areas. The only limit on how yoga can be used to teach a subject is the teacher's ability to be creative.

\subsection{How yoga can be incorporated into the curriculum}

Research has found that one of the greatest barriers to incorporating yoga into a classroom is the limited availability of time during the school day, and the pressure to teach the entire curriculum [6]. Thus, teachers often feel that they do not have time to incorporate additional instruction outside of the curriculum. One way in which, to overcome this barrier is to incorporate yoga into the curriculum; thus circumventing the need to set aside a specific time solely for yoga instruction.

Yoga can fit into the curriculum in a variety of ways. In the primary grades yoga can easily be incorporated into the health and physical education curriculum, social studies and drama curriculum, although it is not limited to those subject areas [9]. In kindergarten, yoga can be included in all four 
aspects of the curriculum [15]. This sections outlines a few concrete examples of how yoga can be incorporated, however, teachers are limited only by their creativity in how they choose to adapt yoga to the curriculum.

Yoga easily fits into the Ontario health and physical education curriculum, as the meditation aspects of yoga help them learn personal skills like self-awareness; and different yoga poses teach movement competence and help children label body parts teaching children about human development [9]. Teaching yoga to young children sets the foundation for active living throughout the child's life. Furthermore, yoga can be used to teach social studies as mindfulness helps children develop a sense of identity. Yoga also teaches children about other cultural practices and emphasizes the importance of respect for others [9, 11]. Yoga can also be incorporated into drama as poses enable children to learn how to use their bodies to represent characters (e.g. cats, bears, snakes) and ideas (e.g. wind, grass, water) [10].

The kindergarten curriculum in Ontario consists of four key components: engagement, belonging, expression, and well-being [15]. The teacher's engagement is a precursor to the student's success. Disengaged teachers produce disengaged students which is detrimental to the atmosphere and culture of a classroom [15]. In yoga it is crucial that teachers participate in the session because modelling the correct poses allows students to understand how to move their bodies.

Fostering a sense of belonging by creating trusting, genuine relationships between teachers and students is crucial for the student's ability to learn [10]. Much of the feedback from the students focused on how much they enjoyed sharing and giving feedback, and how their relationships with others encouraged them to participate and become engaged in the session. If there is not a positive trusting relationship established in the classroom, students will not contribute to their learning [11]. Yoga teaches children how to express themselves by fostering a sense of identity and using movement to convey meaning, and fosters a sense of well-being as they learn to identify and express emotions in addition to improving their ability to focus and learn new information $[2,11]$.

\subsection{Controversy in the literature}

While the benefits of yoga for adults and older teens is well established, there are few studies that focus on the benefits of yoga for children in below the age of 8 [16]. Additionally, studies that examine yoga as an intervention for children with disabilities often take place outside the classroom [4], [6], [16]. Many of the studies done with young children find inconsistent results, or find that yoga is not as beneficial for them as it is to older children and adults [16]. However, the literature on the benefits of teaching yoga to children is fraught with widespread methodological issues including: lack of appropriateness in terms of session duration, the failure to account for confounding variables, and a lack of methodology in terms of what teaching method that is being used, (i.e. how the students are being taught) all of which may account for the inconsistent results seen in the literature [2], [4], [6], [7], [8], [14], [16], [17].

A systematic review of the literature found widespread methodological issues in terms of the appropriate duration of yoga sessions [7], 9], 16]. In this review of the twelve yoga studies that were done in an elementary school setting, more than half asked children to participate in sessions that were at least an hour long, and even one of the studies in a high school asked students to participate in a yoga session that lasted longer than two hours [7]. Other studies noted similar issues in terms of the length of yoga session for children [8]. These studies contradict other literature that suggests that yoga sessions should not last for longer than 45 minutes for children in elementary school or longer than 90 minutes for students in high school [16]. Additionally, it was found that several studies did not disclose how long their yoga sessions were, making these studies impossible to replicate [8], [16].

Several studies that examined the benefits of yoga for children with special needs failed to account for confounding variables: such as additional therapy and child engagement [4], [6], [7], [8], [18].

A study on how yoga can be used to help students with ASD was done in at the same time as Applied Behavioural Analysis therapy; therefore it is difficult to determine if the improvements in mimicry and eye-contact were a result of the therapy of the yoga [4]. Another study done with children with ADHD examined yoga as a way to help these children focus their attention and control their impulsiveness [18]. This study did not find conclusive results however, these children were also taking stimulants to help with the ADHD and failed to control for the effects of the ADHD medication [18].

If students are not engaged, they are not receiving any benefit from the yoga and thus the results of any study reflect how engaged the students were. Despite this, few studies examined the importance of engagement and its impact on their results [6].

Despite the importance of replication is research few studies mentioned how they taught yoga, merely stating that they did yoga poses, breathing or meditation [6], [14], [16], [17]. Additionally, 
different studies defined yoga differently and while many studies included breathing, yoga poses, and mediation, a few studies only focused on meditation, despite still defining it as yoga [14], [17]. This lack of consistency means that it is difficult for researchers to compare or replicate studies. Additionally, without knowing how yoga was taught to the students it is impossible to establish best practice and determine what teaching methodology is the most effective.

This study adds to the existing body of literature on the benefits of teaching yoga to young students by establishing that student engagement and attention is a key confounding variable, which future studies should take into consideration, and establishes the maximum time that children can pay attention. This study also establishes a teaching methodology that can be used to keep students engaged in a yoga lesson and outlines a clear methodology which can be replicated in future research.

\section{Methods}

The population for this study consisted of 20 children between the ages of 4 to 8 who attend a local daycare centre in Sudbury, Ontario. As the research took place over the summer the children were attending full day daycare. The children were previously acquainted with yoga as it had been included in their programming. Before teaching the yoga session, a visit was arranged so the researcher could become familiar with the environment and the children.

The session occurred shortly after lunch and took place indoors due to the weather. Yoga mats were set up in a horseshoe shape, ensuring that all children could clearly see and had enough room to move. The yoga lesson lasted for 30 minutes though an additional fifteen minutes each was given for set up, and to collect feedback after the lesson. The children were absent during the set-up of the classroom, and thus the total time of the session was 45 minutes.

The session started with a five minute breathing exercise, wherein students were instructed to close their eyes and put their finger's in their ears, breathe in for 7 breaths, hold the breath for three seconds and breathe out while humming for seven seconds. Putting their fingers in their ears and closing their eyes helped to block extraneous sensory input, while the humming helped focus their attention inwards. Students were asked to repeat this breath 5 times; followed by fifteen minutes of yoga. The yoga poses were demonstrated before the students were asked to try them. The researcher participated in the session along with the children so they had someone to mimic if they needed help. The students were asked to perform 4 different animal poses and to hold each pose for 1 minute and repeated each pose twice. Animal poses were used as these poses are simple to learn and often enjoyed by children [2].

Animal poses are easier for young children to learn because the poses are more concrete and children can easily guess what the pose will look like based on the name. For example, the snake pose, which consist of lying down on your stomach and lifting your shoulders, neck, and head of the ground is easier for students to remember then a pose such as 'exalted warrior, which is a type of lunge. If children are asked to mimic a snake they will often lie on their bellies and try to slither on the floor, the yoga pose is an extension of that. Furthermore, animal poses are less intimidating for children as they are often less complex and demanding then other poses, this helps children feel more confident and secure during the lesson. Additionally many children with intellectual disabilities, low executive functioning and learning disabilities have difficulty understanding abstract concepts, therefore using concrete imagery facilitates learning for these students.

Students were asked to make animal poses while exhaling to help them focus on their breath, relax and encourage engagement and build confidence. Students were asked to perform the Cat, Cow, Snake and Bear poses; these poses were chosen as they were simple to perform and were easily adaptable to students' needs. Each specific pose strengthens different muscle groups and can improve gross motor skills. The four yoga poses that were used were chosen because they were easy for children to perform and when combined offered a range of stretches that help ease tension due to the demands of a classroom environment.

The Cat and Cow pose helps offset the strain of sitting at a desk for long periods of time, while the Snake pose helps to strengthen core muscles as well as the shoulders, back and neck, the Bear pose improves flexibility and if children walk helps to improve coordination.

To consolidate what they had learned the students were instructed briefly on how to play a game called Bear Hunt. Then students would sing the Bear Hunt song while performing the poses that corresponded to the song. This song was chosen as because of its versatility. The song can easily be expanded on and new verses added, as children learn more poses, or different animals or objects can be used to reflect more challenging poses as children develop their skills.

Games are an appropriate teaching medium for young children as it encourages engagement and children respond better to the lesson [6]. Additionally, games are a way of allowing 
children to challenge each other and be competitive in a non-threatening manner that does not jeopardize the classroom environment. Games are a fun interactive form of play-based learning that allows children to become familiar with the inquiry process, explore and think creatively. Playbased learning forms the foundations of the inquiry process that is central to all subject areas of the Ontario curriculum [10], [15].

To ease into the regular classroom routine after yoga another 5 minutes of breathing combined with stretching took place. Students were instructed to perform the same breath as the one they started with, then tense and release their shoulders, arms, fists, stomach, legs and toes, three times each. By tensing and relaxing each muscle individually students become more aware of their bodies, gradual muscle relaxation is often a technique used to help people who suffer from anxiety and panic attacks as it helps people shift their focus onto present stimuli, and is another form of mindfulness.

The session concluded with 5 minutes of guided meditation where students were told to close their eyes and imagine a balloon floating on a string, being released and floating up into the clouds before finally coming down to earth. At which point they were told to open their eyes. Guided mediation was used as it provides a better focal point for children, is easier for beginners who are unused to meditative practices, and is better suited for children with attention problems [6]. Finally, children were given 15 minutes to provide feedback and share their thoughts on the lesson.

As a result of the brief nature of the feedback given by the children, qualitative methods were used to pull important themes from the feedback. Themes were categorized based on how they related to the guidelines for early childhood education, and according to what the children thought was the most important part of the lesson.

\section{Analysis of Findings}

All the children could successfully complete the Cat13Cow and Snake poses though three children had difficulty differentiating between flexing and arching their back. One child could not complete the Bear pose in a standing position and had to kneel in order to touch the floor. Most of the children could accurately guess what the poses would look like based on the names of the animals.

The most important finding concerned the attention span and appropriateness of the length of the session. In the beginning of the session 18 out of 20 children chose to participate and preformed the poses. All of the children participated in the game however, during the breathing and stretching exercises five children began fidgeting and were off topic. Once the guided mediation session was complete nine of the children had lost their concentration. While giving feedback thirteen of the children had lost interest and had difficulty sitting still (see Figure 1).

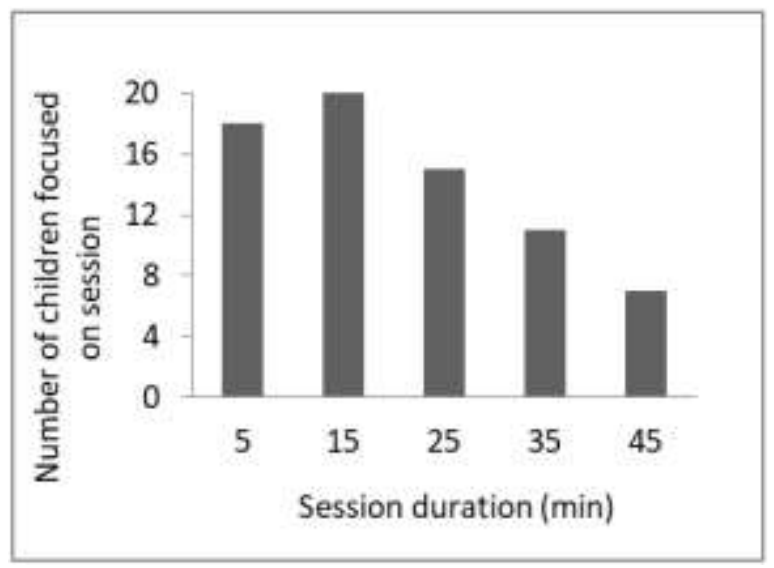

Figure 1. Students' attention spans over the duration of the session

When asked to provide feedback after the lesson children commented on the following key elements: engagement and space. These key themes address the barriers for teaching yoga in a classroom setting and illustrate the importance of appropriate teaching methods

Engagement: Seventeen children commented that the lessons were fun, that they enjoyed the game, or enjoyed making animal noises. These finding coincide with other research that remarks on the importance of games and play to keep students engaged in the lessons [6]. Many of the studies are not complete in their methodology, and merely outline the length and duration of the yoga session without explaining how they taught the session.

Furthermore, the studies make no mention of how engaged the students were, without this information it is impossible to tell if the lack of consistency in these studies is because yoga is less effective with young children, or if the lack of consistency is because the children were not engaged in the lesson.

This study controlled for engagement as a confounding variable by ensuring that all of the children were engaged during the game. If children are not taught yoga in an appropriate manner, for example if the instructor does not include games or concrete examples, to ensure the children understand and are engaged in the lesson, children will not participate and therefore the study will not have consistent outcomes. 
Space: "Everyone needs their own 'bubble' a quiet space to focus and when you're near something you like you focus better." - A comment from one of the children after teaching a lesson. Six children out of 20 commented on how important it was to have their own space, or how they needed 'room to move', or how having their own space made it easier to focus.

Each child was given a puzzle mat, which they could sit on, and without being told the children knew not to go onto each other's mats. This demonstrates that finding a way to create a separate space for each child is crucial to teaching yoga, and that children must be able to recognize the boundaries of their space and another student's space. Younger children need a more visible barrier then older children do, mats, or hula hoops are one way of establishing these boundaries clearly. Having enough space to move in a classroom is important as it helps the students focus on the lesson.

Previous studies found mixed result for the benefits of yoga when taught to young children; this may be due the failure to provide space for children in a way that they recognize [6]. If children were not given any way to determine which space was theirs, or not provided with any instructions on making sure they had enough room; it will impact their ability to focus on and therefore benefit from the lesson, leading to a lack of results in the study [6].

\section{Discussion}

The literature has established that, yoga is a beneficial tool to teach children emotional and attention regulation, improves the outcomes of children with mood disorders and ADHD, helps children with Autism learn social skills and improves overall academic performance for all students [1], 2], [3], [5], [11]. Yoga can be incorporated into the curriculum and the main barriers that prevent teachers from bring yoga into their classrooms can be overcome by creativity, games and innovative teaching [6], [15], [17].

However, there is a lack of consistency regarding the benefits of yoga in young children in early primary school, kindergarten and prekindergarten [16,]. This may be due to the lack of age appropriate methodology and teaching methods used by many of the studies [2], [4], [6], [7], [14, [16, [17]. It is important to establish an appropriate methodology because young children will not receive benefits from yoga if they are unable to focus on the lesson or understand the lesson because they are not being taught in a style that is conducive to their understanding [6]. If children cannot focus, or understand the lesson then they are not engaged, and their failure to engage in the lesson means that they will not benefit. This lack of engagement and the lack of impact from yoga create inconstancies in much of the existing literature.

This study contributes to the existing research by establishing the appropriate length of a session by determining the optimal session length needed to ensure students can focus and be engaged in the lesson. This study also outlines a methodology that maintains engagement and teaches yoga in a format that young children can understand.

Yoga does not have to be taught independent of the curriculum; this merely adds to the workload of both teachers and students reducing the amount of time available in the day. Instead, it is more productive if yoga can be integrated into the curriculum and combined with other teaching expectations. The lesson incorporated curriculum expectations from three different subjects: health and physical education, social studies, and drama [10].

For the duration of the guided meditation children began to lose focus and talk with each other (see Figure. 1). Many students had difficulty following the meditation as the session started to drift off topic, and the amount of fidgeting increased towards the end of the meditation, around 25 minutes into the session. This is a key methodological finding as it demonstrates that much of the previous research was not effective as the yoga sessions were not of an appropriate length for young children. This study concludes that yoga sessions should be no more than 30 minutes, as that appeared to be the upper limit of their attention span. Once students cease paying attention they stop learning, can disrupt the learning of other's and may start displaying behavioral problems. At which point the teacher loses instructional time to classroom management and the children no longer receive the benefit from the yoga.

All of the students provided feedback on how silly the animal noises were, or how much they liked the song. This speaks to the appropriateness of using concrete examples, and songs to make yoga engaging [2].

\section{Conclusion}

Yoga is extremely versatile, poses can be adapted if a child is incapable of doing certain pose, some poses are more difficult and so teachers can introduce new poses when they feel their students are capable of doing them.

Different aspects of yoga can have different benefits to different types of students. Students who have difficulties with emotional regulation will benefit substantially from deep breathing as it 
can help children calm down when they are experiencing upset.

The meditation aspect of yoga is beneficial for students with attention deficit disorder, autism or depression as it can help concentration and focus; and will teach children mindfulness which can foster empathy and resilience. Deep breathing can also help children with asthma or other breathing difficulties. Teaching children yoga is not only beneficial to their physical development, it is also crucial for their emotional development and overall wellbeing. Integrating yoga into the health and physical education curriculum helps children develop emotionally, physically and socially, and benefits children mental health.

Teaching children yoga also increases their physical literacy as they learn different movements and start applying them in other activities, as children gain confidence the difficulties of the yoga poses can be increased to suite the individual needs of the children. Yoga also can be incorporated into the social studies curriculum as it helps children develop a sense of identity, improves interpersonal relationships and teaches children about different cultural traditions. Teaching yoga to children not promotes a lifetime habit of physical activity, encourages child development, and teaches children the skills they need to become resilient adults.

A few limitations exist in this study, mainly due to the age of the population. As many students were learning their letters or just beginning to read they were not asked to fill out any questionnaires or to perform a pre and post-tests. Additionally, engaging in such tests would have extended the length of the session and students would have lost focus and become disengaged. For these same reason, interviews were not conducted either as they would have been conducted immediately after the lesson, extending the period of time that students were asked to concentrate. Young children often do not give detailed answers to interview questions and feedback was usually brief and often consisted of variations of the phrase "it was fun".

There were time restraints due to the nature of the study and thus the number of sessions was limited to a single lesson. Furthermore, only the Ontario curriculum was explored and yoga may be more difficult to incorporate into other curriculums.

In conclusion, this study has highlighted the inadequate methodologies used in much of the existing research, as yoga sessions over 30 minutes in length are less effective for young children [2], [4], [5], [7], [9]. Therefore, additional research needs to be done using shorter session duration to determine the best practises for teaching yoga to this age group.

\section{References}

[1] Avashalom, S., Bar-Dov, A., and M. Ehud, (2010). Here and Now: Yoga in Israeli Schools. International Journal of Yoga, 3, pp. 42-47.

[2] Bergen-Cico, D., Raymond, K., and R.A. Razza, (2013). Enhancing Preschoolers' Self-Regulation Via Mindful Yoga. Journal of Child and Family Studies, Springer Science and Buissness Media, Syracuse NY, 24(2), pp.372-385.

[3] Copeland-Linder, N., Perry-Parrish, C., Siblings E., and Webb, L., (2016). Mindfulness-Based Approaches for Children and Youth. Current Problems in Pediatric and Adolescent Health Care, Mosby Inc., 46(6) pp. 172178.

[4] Porter, J., (2012). Yoga as an Effective Behavioural Intervention for Children Diagnosed with an Autism Spectrum Disorder. Graduate Annual, Philadelphia, USA, 1(9), pp. 25-30.

[5] Satheeshkumar, K. and Rajaguru, S., (2016). Impact of Yogic Practice on Adjustment Behaviour of School Children. International Education and Research Journal, 2(4), pp. 17-18.

[6] Harssar, J., (2017). How Teachers are Using Mindfulness as Responsive Pedagogy for Students with ADHD. Thesis Dissertation, Ontario Institute for Studies in Education, University of Toronto, Toronto, Ontario.

[7] Butzer, B., and Khalsa, S.B.S., (2016). Yoga in School Settings: A research review. Annals of the New York Academy of Sciences, New York Academy of Sciences, New York, 1373, pp. 45-55.

[8] Feitoza, J. M., Ferreira-Vorkapic, C., Kozasa, E., Marchioro, M., Simões, J., and Telles, S., (2015). "Are there benefits from Teaching Yoga at Schools?" A Systematic Review of Randomized Control Trials of Yoga-Based Interventions. Evidence-Based Complementary and Alternative Medicine, Hindawi Publishing Co., pp. 1-17.

[9] White, L.S., (2009). Yoga for Children. Pediatric Nursing, Coimbatore India, 35(5), pp. 277-295.

[10] Ontario Ministry of Education, (2016). Grade 1 Curriculum Documents. The Ontario Curriculum: Elementary, Queen's Printer for Ontario, Ontario Canada.

[11] Butterfield, N., Schultz, T., Rasmussen, P., Proeve, M., (2017). Yoga and mindfulness for anxiety and depression and the role of mental health professionals: a literature review. The Journal of Mental Health Training, Education and Practice, 12(1), pp. 44 - 54

[12] Chou, C. C., and Huang, C. J., (2017). Effects of an 8 -week yoga program on sustained attention and discrimination function in children with attention deficit hyperactivity disorder. Peer Journal, 5, e2883. 
[13] Thomas, L., (2008). Being Present: Mindfulness and Yoga at Westminster Center School. Horace, 24(2), $\mathrm{n} 2$.

[14] Albrecht, N., Albrecht, P., and Cohen, M., (2012). Mindfully teaching in the classroom: A literature review. Australian Journal of Teacher Education, 37(12), 1-14.

[15] Ontario Ministry of Education, (2016). The Kindergarten Program. Queen's Printer for Ontario, Ontario Canada, pp. 46-92.

[16] Kaley-Isley, L. C., Peterson, J., Fischer, C., and Peterson, E. (2010). Yoga as a complementary therapy for children and adolescents: a guide for clinicians. Psychiatry (Edgmont), 7(8), 20.

[17] Francis, C., (2017). 'On-The-Spot' Mindfulness Based Practices for Addressing Behavior Challenges among 3rd Graders. Master's Projects and Capstones. 609.

[18] Lange, K. M., Makulska-Gertruda, E., Hauser, J., Reissmann, A., Kaunzinger, I., Tucha, L., and Lange, K. W. (2014). Yoga and the therapy of children with attention deficit hyperactivity disorder. Yoga \& Physical Therapy, 4(3), 1000168. 